\title{
Arbeit und Suizid
}

\section{Erhard Taverna}

Mitglied der Redaktion

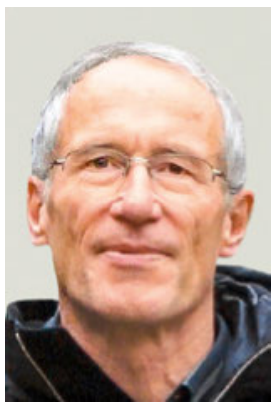

In Artikel 23 und 24 beschreibt die Allgemeine Erklärung der Menschenrechte von 1948 nicht nur das Recht auf Arbeit, sondern auch grundlegende Rechte in der Arbeitswelt. Fünfzig Jahre später erweitert die Europäische Sozialcharta diese Forderung um das Recht auf eine Wohnung. Da die Schweiz die Charta nicht unterzeichnet hat, führt AvenirSocial seit 2009 eine Kampagne "Pro Sozialcharta», um diese Rechte auch hierzulande durchzusetzen.

Deklarationen und Gesetze sind das eine, die reale Wirtschaftspolitik das andere. Zahlreiche Untersuchungen belegen, dass Arbeitslosigkeit Menschen in den Suizid treiben kann. Gemäss der Studie in der Zeitschrift The Lancet Psychiatry online kommt eine Arbeitsgruppe der Psychiatrischen Universitätsklinik Zürich 2015 zum Schluss, dass jeder fünfte Suizid weltweit in einem Zusammenhang mit Arbeitslosigkeit steht. Die sorgfältigste und meistzitierte Studie The Impact of fiscal austerity on suicide mortality: Evidence across the Eurozone periphery ist ein Gemeinschaftswerk der Universitäten Webster und Portsmouth, veröffentlicht im gleichen Jahr in Social Science \& Medicine. Es ist die erste systematische Arbeit zum Ländervergleich von Griechenland, Irland, Italien, Portugal und Spanien für die Jahre 1968-2012. Die Folgen

\section{Zahlreiche Untersuchungen belegen, dass Arbeitslosigkeit Menschen in den Suizid treiben kann.}

der Sparpolitik werden unter Berücksichtigung ökonomischer und soziodemografischer Unterschiede, aufgeschlüsselt nach Geschlecht und Alterskohorte, auf kurz-, mittel- und langfristige Auswirkungen untersucht. Eine trocken zu lesende Materie, die, beruhend auf einer soliden Datengrundlage, mit Tabellen und Diagrammen prozentgenau die tödlichen Zusammenhänge statistisch erfasst. Im dritten Jahr nach Beginn des Sparprogramms steigt die Kurve dramatisch nach oben. In Griechenland muss man von einer Epidemie sprechen, bei der 9,1\% der Suizidfälle Menschen über
60 Jahre betrifft, die Pensionen und Gesundheitsversicherungen teilweise oder ganz verlieren. Im vierten Jahr wächst der Anteil an Suiziden der über Vierzigjährigen, überwiegend alleinstehenden Langzeitarbeitslosen. In Irland ist auch, nach erneutem Anstieg des Wirtschaftswachstums, die dramatisch gestiegene Selbstmordrate nicht zurückgegangen. Wo das soziale Netz zerstört wurde, ist der Konjunkturaufschwung nicht mehr angekommen.

Fazit für alle Länder: Wo der Staat keine angemessen funktionierende Sozial- oder Gesundheitsinfrastruktur anbieten kann oder will, führt die Finanzkrise zu einschneidenden, gesellschaftlichen Veränderungen mit

\section{Frauen und Männer, die mit ihrer Arbeit das soziale Umfeld und vielleicht per Zwangs- räumung auch noch die Wohnung verlieren, werden psychisch krank.}

dem Verlust zahlreicher Menschenleben. Es dürften psychisch gefährdete Menschen sein, die als Erste ihren Job verlieren und vergeblich nach Hilfe suchen. Denkt man an die Folgen für Angehörige oder an die Zahl der arbeitslosen Jugendlichen, werden die Langzeiteffekte dramatisch. Erzwungene Migration, Kriminalität und verlorene Lebensjahre in einer chancenlosen Umgebung dürften über Generationen hinaus nachwirken. Frauen und Männer, die mit ihrer Arbeit das soziale Umfeld und vielleicht per Zwangsräumung auch noch die Wohnung verlieren, werden psychisch krank. Da kann der Suizid bald einmal als einziger, selbstbestimmter Ausweg erscheinen. Hartz IV sei Mord per Gesetz, ist in Deutschland häufig zu hören.

Die Medikalisierung von Gesellschaftsproblemen lässt oft leicht vergessen, dass der Eigennutz der Politiker und Abstimmenden das Elend von Mitbürgern und Mitbürgerinnen verursachen kann. Charles Fournier (1772-1837) verband seine Kapitalismuskritik mit der Forderung nach einem bedingungslosen Grundeinkommen. Seine Sprache ist veraltet, aber seine Ideen wirken bis heute nach. 